\title{
Herzschrittmacher- und ICD-Infektionen
}

\section{Pacemaker and ICD Infections}

\author{
Autoren \\ Bernd Nowak, Felix Weise, Oliver Przibille \\ Institut \\ CCB, Agaplesion Bethanien Krankenhaus, Frankfurt a. M.
}

\section{Schlüsselwörter}

Herzschrittmacher, ICD, Infektion, Explantation

Key words

pacemaker, ICD, infection, explantation

\section{Bibliografie}

DOI https://doi.org/10.1055/a-0681-6383

Aktuel Kardiol 2018; 7: 453-457 @ Georg Thieme Verlag KG Stuttgart · New York | ISSN 2193-5203

\section{Korrespondenzadresse}

Prof. Dr. med. Bernd Nowak

CCB Cardioangiologisches Centrum Bethanien,

Agaplesion Bethanien Krankenhaus

Im Prüfling 23, 60389 Frankfurt a. M.

Tel.: 069/94502 80, Fax: 069/461613

b.nowak@ccb.de

\section{ZUSAMMENFASSUNG}

Die Infektion von Herzschrittmachern und implantierbaren Defibrillatoren stellt ein zunehmendes Problem dar, da immer mehr komplexe Systeme bei multimorbiden Patienten implantiert werden. Wesentlich sind die Kenntnis der Risikofaktoren für eine Aggregatinfektion und die konsequente Präven- tion, u. a. durch eine perioperative Antibiotikaprophylaxe. Die Diagnostik kann, gerade bei latenten Infektionen, schwierig sein. Sie umfasst neben Blutkulturen die transthorakale und transösophageale Echokardiografie zur Abklärung von Vegetationen der Elektroden und/oder einer Endokarditis. In aller Regel ist neben einer verlängerten Antibiotikagabe die komplette Systemexplantation erforderlich. Die Indikation zur Reimplantation muss kritisch überprüft werden. Diese erfolgt nach vollständiger Ausheilung sofern möglich kontralateral. Alternativen können eine epikardiale Implantation oder elektrodenlose Systeme sein.

\section{ABSTRACT}

Infection of pacemakers and implantable defibrillators has been becoming an increasing problem as more and more complex systems are implanted in multimorbid patients. Essential are the knowledge of the risk factors for a device infection and the consistent prevention, i.a. by a perioperative antibiotic prophylaxis. The diagnosis can be difficult, especially with latent infections. In addition to blood cultures, diagnostics include transthoracic and transesophageal echocardiography in search of vegetations on the leads and/ or an endocarditis. As a rule, in addition to a prolonged antibiotic administration, the complete system explantation is required. The indication for reimplantation must be critically reviewed. This is done, after complete healing, if possible contralaterally. Alternatives may be an epicardial implantation or leadless systems.

\section{Einleitung}

Die Infektion von Herzschrittmachern und implantierbaren Defibrillatoren (cardiac implantable electronic devices, CIED) stellt ein schwerwiegendes Problem dar. Für eine adäquate Therapie müssen neben einer Antibiotikagabe in der Regel Aggregat und Elektroden vollständig explantiert werden. Aggregat- und/oder Elektrodeninfektionen gehen mit einer erhöhten Morbidität und Mortalität einher. Darüber hinaus verursachen sie erhebliche Folgekosten für das Gesundheitswesen [1].

CIED-Infektionen stellen eine zunehmende Komplikation der Aggregattherapie dar, sie steigen im Vergleich zur Implantationshäufigkeit überproportional an [2-4]. Dies erklärt sich durch die Zunahme komplexerer Systeme, insbesondere CRT-Schrittmacher und CRT-Defibrillatoren. Darüber hinaus werden häufiger multimorbide Patientinnen und Patienten versorgt, bei denen bereits die Begleiterkrankungen ein höheres Infektionsrisiko zur Folge haben. Eine große dänische Kohortenstudie mit 46299 Schrittmacherimplantationen und einer Nachbeobachtung über 236888 Patientenjahre ergab für frühe Infektionen (innerhalb eines Jahres nach Implantation) eine Rate von 4,82/1000 Schrittmacherjahre bei Erstimplantationen und von 12,12/1000 Schrittmacherjahre für Aggregatwechsel [5]. Späte Infektionen nach mehr als einem Jahr traten bei Neuimplantationen in 1,02/1000 bzw. bei Aggregatwechsel in 3,26/1000 Patientenjahre auf. Bei komplexeren Systemen liegen die Infektionsraten höher: bei implantierbaren Defibrillatoren (ICD) beträgt sie bei Neuimplantation 0,7 bis $1,2 \%$ und bei kardialen Resynchronisationssystemen (CRT) 1,7 bis 9,5\% [6]. 


\section{WAS IST WICHTIG?}

- Aggregatinfektionen stellen ein zunehmendes Problem dar und sind mit einer erhöhten Morbidität und Mortalität behaftet.

- Die wirksamste Therapie ist eine Kenntnis der Risikofaktoren und eine konsequente Prävention. Eine perioperative Antibiotikaprophylaxe ist erforderlich.

- Hämatome und frühe Reoperationen stellen ein wesentliches Risiko für einen Aggregatinfekt dar.

- Die Behandlung erfordert neben einer verlängerten Antibiotikagabe in der Regel die komplette Systemexplantation.

- Seltene Sonderfälle sind zum einen ein kleiner Nahtabszess früh nach Implantation sowie zum anderen ein Versuch der Aggregatrettung, wenn eine Explantation nicht infrage kommt.

Nach Aggregatwechseln oder Aufrüstungen sind 2- bis 4-fach höhere Infektionsraten zu erwarten.

Im deutschen Herzschrittmacher- und Defibrillatorregister wurden 2015 über 18000 Revisionsoperation wegen Aggregatproblemen dokumentiert [7]. Hierbei entfielen 9 bis 11\% auf $\mathrm{Ag}$ gregatinfektionen und Taschenperforationen. Bei letzteren ist meist auch von einer infektiösen Ursache auszugehen ( $\triangleright$ Tab. 1). Auch nach erfolgreicher Therapie einer CIED-Infektion bleibt das Mortalitätsrisiko über mindestens 3 Jahre bei den betroffenen Patientinnen und Patienten im Vergleich zu solchen ohne Infektion signifikant erhöht:

- Herzschrittmacher: 53,8 vs. $33 \%(p<0,001)$,

- ICD: 47,7 vs. $31,6 \%(p<0,001)$ und

- CRT-D: 50,8 vs. $36,5 \%(p<0,001)$ [8].

Dies unterstreicht, dass CIED-Infektionen als schwerwiegendes Problem anzusehen sind.

Eine Endokarditisprophylaxe ist bei Patienten mit implantiertem Herzschrittmacher oder ICD jedoch nicht erforderlich.

\section{KURZGEFASST}

Aggregatinfektionen treten aufgrund komplexerer Systeme und multimorbider Patienten zunehmend auf, wobei Aggregatwechsel besonders gefährdet sind. Folge ist eine deutlich erhöhte Mortalität.

\section{Prävention}

Die beste Therapie einer CIED-Infektion ist deren konsequente Prävention. In verschiedenen Arbeiten konnten Risikofaktoren, die mit einer erhöhten Infektionsrate einhergehen, identifiziert werden $[1,3,6,9]$. Zu den nicht beeinflussbaren Risikofaktoren gehören z. B. eine Niereninsuffizienz insbesondere bei chronischer Dialysebehandlung, ein Diabetes mellitus oder das männliche Geschlecht sowie jüngeres Patientenalter. Weitere Risikofaktoren,
- Tab. 1 Neuimplantationen, Aggregatwechsel und Revisionen wegen Taschenproblemen in Deutschland (2015).

\begin{tabular}{|c|c|c|c|c|}
\hline & \multicolumn{2}{|c|}{$\begin{array}{l}\text { Herzschritt- } \\
\text { macher }\end{array}$} & \multicolumn{2}{|l|}{ ICD } \\
\hline Neuimplantationen & \multicolumn{2}{|c|}{75730} & \multicolumn{2}{|c|}{30002} \\
\hline Aggregatwechsel & \multicolumn{2}{|c|}{18725} & \multicolumn{2}{|c|}{10078} \\
\hline $\begin{array}{l}\text { Revisionsoperationen we- } \\
\text { gen Taschenproblemen }\end{array}$ & \multicolumn{2}{|c|}{8344} & \multicolumn{2}{|c|}{9835} \\
\hline - Infektion & 675 & $8,1 \%$ & 714 & $7,6 \%$ \\
\hline - Taschenperforation & 286 & $3,4 \%$ & 153 & $1,6 \%$ \\
\hline $\begin{array}{l}\text { - sonstiges Taschen- } \\
\text { problem }\end{array}$ & 717 & $8,6 \%$ & 460 & $4,9 \%$ \\
\hline
\end{tabular}

welche zumindest partiell beeinflusst werden können, sind z.B. der Einsatz passagerer transvenöser Schrittmacher, ein Taschenhämatom, Fieber oder vorbestehende systemische Infekte. Es ist unklar, ob bei dringlicher Implantationsindikation und einer aktiven Infektion eine passagere Stimulation oder eine primäre permanente Implantation zu bevorzugen sind. Meist wird zunächst passager stimuliert, mit der Folge eines wiederum erhöhten Risikos für eine Aggregatinfektion.

Je komplexer die implantierten Systeme, desto höher ist generell das Infektionsrisiko [10]. Das gleiche gilt für frühe Reoperationen. Diese sollten daher wenn möglich vermieden werden. Aggregatwechsel und Aufrüstungen sind ebenfalls mit einem erhöhten Infektionsrisiko verbunden. Die einzelnen Risikofaktoren für eine CIED-Infektion mit der jeweiligen relativen Risikoerhöhung sind in > Tab. 2 aufgeführt.

Durch eine Reihe präoperativer Maßnahmen kann das Infektionsrisiko gesenkt werden [9]: Vorbestehende Infektionen müssen suffizient behandelt sein. Im geplanten Operationsfeld dürfen keine EKG-Elektroden angebracht werden, um Hautschädigungen zu vermeiden. Genauso muss die Haarentfernung im Operationsfeld mit einer Haarschneidemaschine und nicht mit einem scharfen Rasierer erfolgen, um Hautverletzungen als potenzielle Infektionsquellen zu vermeiden. Zentrale Venenkatheter und Drainagen sollten mindestens 24 Stunden präoperativ entfernt werden, sofern dies medizinisch vertretbar ist.

Die perioperative Antibiotikagabe muss standardmäßig durchgeführt werden $[3,5,6,9]$. In einer prospektiven randomisierten, doppelblinden und placebokontrollierten Studie wurde die unmittelbar präoperative Gabe von $2 \mathrm{~g}$ Cefazolin i.v. untersucht [11]. Die Studie wurde bereits nach Einschluss von $649 \mathrm{~Pa}-$ tienten (bei geplanten 1000 Patienten) vorzeitig abgebrochen, da die Rate an Aggregatinfektionen nach 6 Monaten durch die Antibiotikagabe von 3,28 auf 0,64\% signifikant gesenkt wurde.

Bei der perioperativen Antibiotikaprophylaxe sollte der Spitzenspiegel im Blut bei Operationsbeginn erreicht sein. Empfohlen wird z. B. die Gabe eines Cephalosporins der 1. Generation, z. B. $2 \mathrm{~g}$ Cefazolin 1 Stunde präoperativ. Bei MRSA-Besiedelung oder einer Cephalosporinallergie kann z. B. Vancomycin $20 \mathrm{mg} / \mathrm{kgKC}$ 2 Stunden präoperativ verabreicht werden $[12,13]$. Eine umfassende Empfehlung der British Heart Rhythm Society empfiehlt 
- Tab. 2 Risikofaktoren für Aggregatinfektionen (mod. nach [3, 6, 9]).

\begin{tabular}{|l|l|}
\hline Risikofaktor & relatives Risiko \\
\hline Niereninsuffizienz & 1,3 \\
\hline Dialyse & 8,6 \\
\hline chronische Hauterkrankung & 10,6 \\
\hline chronisch obstruktive Lungenerkrankung & $1,2-9,8$ \\
\hline Diabetes mellitus & 2,3 \\
\hline männliches Geschlecht & 1,5 \\
\hline jüngeres Alter & $1,4-4,5$ \\
\hline Kortisonbehandlung & 13,9 \\
\hline Erfahrung des Operateurs (<11 vs. $>29 / J a h r)$ & 2,5 \\
\hline Taschenhämatom & $4,0-6,7$ \\
\hline passagerer Schrittmacher & $2,5-5,0$ \\
\hline Fieber/systemische Infektion & 5,8 \\
\hline komplexe Systeme & $1,3-5,4$ \\
\hline frühe Reoperation & $2,7-15$ \\
\hline Aggregatwechsel & 2,7 \\
\hline früherer CIED-Infekt & 11,3 \\
\hline Anzahl vorangegangener CIED-Operationen & $2,7-8,7$ \\
\hline hoher Charlson-Komorbiditäts-Index & $2,7-3,0$ \\
\hline & \\
\hline
\end{tabular}

die generelle Gabe eines Glykopeptides (Teicoplanin oder Vancomycin), wobei eine Kombination mit Gentamicin kontrovers diskutiert wird [12]. Für eine Applikation von Antibiotika oder Antiseptika direkt in die Aggregattasche besteht keine Evidenz.

Intraoperativ ist eine suffiziente Blutstillung entscheidend, da ein Taschenhämatom einen wesentlichen Risikofaktor für eine Aggregatinfektion darstellt $[3,11]$. Sollte es zu einem Hämatom kommen, ist eine Punktion kontraindiziert, da diese eine Infektion zusätzlich begünstigt! Auch eine Reoperation sollte hierbei möglichst vermieden werden, da diese das Infektionsrisiko wiederum erhöht [12]. Bei schlanken Patienten muss auch bei einer Schrittmacherimplantation eine subpektorale Aggregatlage erwogen werden. Ob im Rahmen eines Aggregatwechsels eine Entfernung der alten Aggregatkapsel Infektionen vermeidet, ist umstritten [6]. Das gleiche gilt für die postoperative Anlage eines Druckverbands über der Aggregattasche.

Eine neue Entwicklung stellt die mit Minocyclin und Rifampicin beschichte Aggregathülle TYRX ${ }^{\circledR}$ dar. In einer Single-Center-Beobachtungsstudie bei 1124 Hochrisikopatienten konnte durch die resorbierbare Hülle die Häufigkeit von Aggregatinfekten innerhalb von 300 Tagen nach Implantation gegenüber 3,1\% in der Placebogruppe auf 0 reduziert werden [14]. Prospektiv randomisierte Daten fehlen derzeit, die WRAP-IT-Studie mit über 7000 Patienten wird voraussichtlich im September 2018 abgeschlossen werden (NCT02277990). In Anbetracht des extrem hohen Preises wird die Verwendung einer antibiotikabeschichten Aggregathülle derzeit Einzelfällen vorbehalten bleiben.

\section{KURZGEFASST}

Die Prävention von CIED-Infektionen ist entscheidend. Taschenhämatome und frühe Reoperationen stellen wesentliche Risikofaktoren dar. Eine perioperative Antibiotikaprophylaxe ist immer erforderlich.

\section{Diagnostik}

Grundsätzlich wird zwischen frühen Infektionen, die innerhalb des 1. Jahres nach Implantation auftreten, und späten Infektionen nach mehr als einem Jahr unterschieden. Etwa 60 bis $80 \%$ der Aggregatinfektionen werden durch Staphylokokken verursacht: frühe Infektionen durch Staphylococcus aureus und späte durch Staphylococcus epidermidis [4,13]. Gram-positive Keime (Corynebakterien, Propionibakterien) und Pilze spielen demgegenüber eine untergeordnete Rolle [3,9]. In etwa 15\% der Fälle sind die Kulturen negativ. Entscheidend für eine Aggregatinfektion ist die Bildung eines Biofilms auf der Implantatoberfläche, welcher Bakterien vor der körpereigenen Abwehr und vor Antibiotika schützt und damit die Behandlung erschwert $[9,13]$.

Ein Aggregattascheninfekt ist die häufigste Manifestation einer CIED-Infektion. Allerdings darf man nie davon ausgehen, dass die Infektion auf die Tasche begrenzt ist, sondern muss immer von einer Ausbreitung entlang der Elektroden, mit dem Risiko einer Endokarditis ausgehen. Die klinische Symptomatik kann sehr variabel sein und ist insbesondere bei schleichenden Infekten oft nur gering vorhanden. Bei frühen Infektionen stehen die klassischen Infektionszeichen Rötung, Schmerz, Schwellung sowie Nahtdehiszenzen im Vordergrund ( $\bullet$ Abb. 1). Späte Infektionen machen sich häufig durch eine Taschenerosion bei geringer klinischer Symptomatik bemerkbar und gehen gehäuft mit intrakardialen Vegetationen einher [15]. Eine erodierte Aggregattasche ist dabei immer als infiziert anzusehen ( $\mathbf{A b b}$. 2)! Alle Aggregatinfekte können aber auch klinisch weitgehend asymptomatisch oder mit unspezifischen Zeichen verlaufen. Eine septischer Schock tritt in weniger als $10 \%$ der Patienten ein [12]. CIED-Infektionen können auch Ausgangspunkt sekundärer Infektionen sein, die z. B. pulmonal oder spinal auftreten.

Jede Veränderung der Aggregattasche wie Rötung, Schwellung, Schmerzhaftigkeit oder Verklebung der Tasche mit eingeschränkter Aggregatbeweglichkeit müssen, genauso wie unklares Fieber oder Infektionszeichen, an eine Aggregatinfektion denken lassen. Neben einer Kontrolle der Entzündungswerte müssen optimal mindestens 2-3 Blutkulturen mit mehr als 6 Stunden Abstand abgenommen werden, insbesondere vor Beginn einer Antibiose. Eine transthorakale und transösophageale Echokardiografie sind erforderlich. Es muss geklärt werden, ob Vegetationen auf den Elektroden vorhanden sind und ob eine Endokarditis mit Klappenvegetationen oder kardialen Abszessen vorliegt. Das transösophageale Echokardiogramm ermöglicht jedoch keine eindeutige Unterscheidung zwischen Thromben und entzündlichen Vegetationen. Auflagerungen an den Elektroden müssen daher immer im klinischen Gesamtkontext bewertet werden $[13,16]$. Sie stellen alleine, ohne sonstige Hinweise auf eine Infektion, keine Indikation zur Systementfernung dar. 


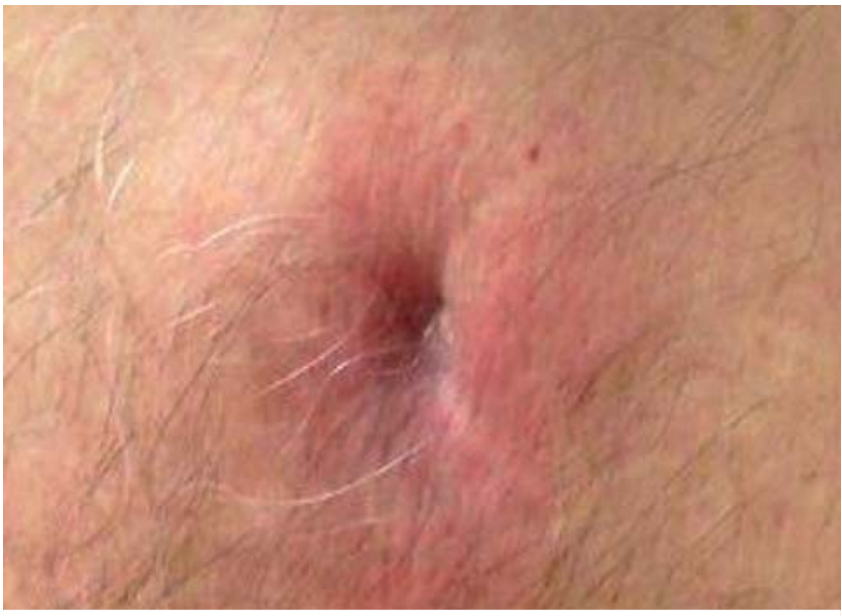

- Abb. 1 Rötung und Einziehung über der Aggregattasche als Zeichen einer Tascheninfektion.

Kann die Diagnose einer Aggregattascheninfektion oder Sondenendokarditis durch diese Untersuchungen jedoch weder gesichert noch ausgeschlossen werden, kommen Spezialuntersuchungen zum Einsatz. Das FDG-PET/CT hat insbesondere für die Tascheninfektion eine hohe Sensitivität und Spezifität (87 und $100 \%$ ). Bei reinen Sondenendokarditiden liegen diese jedoch mit 31 und 62\% deutlich niedriger [17]. Eine Leukozytenszintigrafie mit Tc-99m-HMPAO kann die diagnostische Ausbeute in unklaren Fällen verbessern [18]. Eine Punktion der Aggregattasche darf wegen der Gefahr der bakteriellen Kontamination einer bislang nicht infizierten Tasche nicht durchgeführt werden!

\section{KURZGEFASST}

60-80\% der Aggregatinfektionen werden durch Staphylokokken verursacht. Das klinische Bild kann variabel sein. Entzündungswerte, Blutkulturen, transthorakales und transösophageales Echo gehören zur Basisdiagnostik.

\section{Therapie}

Die entscheidende Therapie bei Schrittmacher- und ICD-Infektionen ist, neben der Antibiotikatherapie, die komplette Explantation von Aggregat und Elektroden [4,6,9,13]. Partielle Operationen wie Aggregatexplantation und Kappung der Elektroden sind fast zwangsläufig mit einem Rezidiv verbunden [6]! Bei Aggregaten, die kürzer als ein Jahr implantiert sind, lassen sich die Elektroden meist ohne den Einsatz spezieller Extraktionswerkzeuge entfernen. Bezüglich der verschiedenen Extraktionstechniken bei chronisch implantierten Elektroden sei auf die aktuellen Empfehlungen der Heart Rhythm Society verwiesen [19]. Sofern Vegetationen vorliegen, die größer als $2 \mathrm{~cm}$ sind, ist eine chirurgische Explantation aufgrund des Embolierisikos erforderlich. Im Rahmen der Explantation sind auch Gewebeproben der Tasche zur bakteriologischen Untersuchung zu entnehmen. Blutkulturen sind erneut 48 bis 72 Stunden nach Explantation abzunehmen.

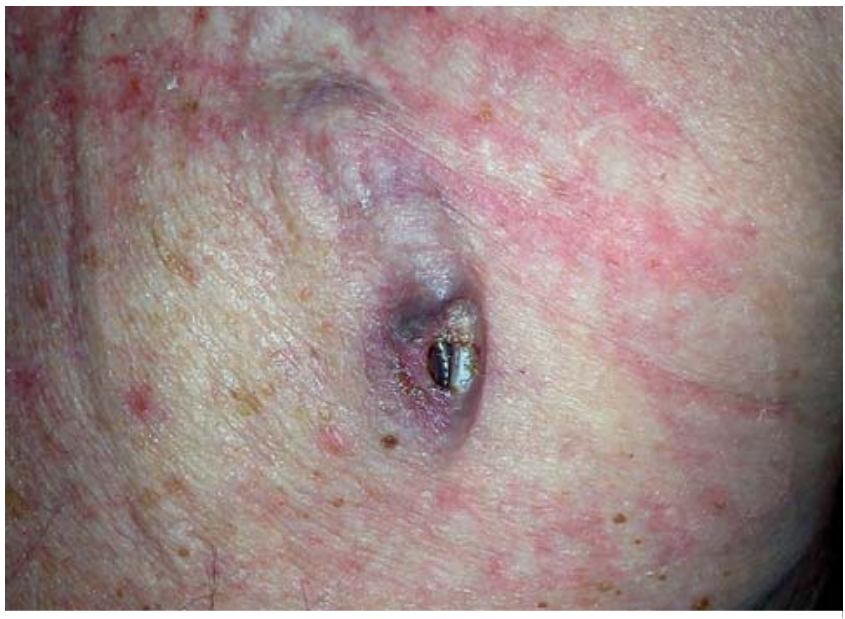

Abb. 2 Perforierte Aggregattasche. Diese ist immer infiziert.

Eine Antibiotikatherapie wird bei einem Tascheninfekt für 1014 Tage, bei einer Bakteriämie für 14 Tage und bei einer Endokarditis für 4 bis 6 Wochen empfohlen. Die Auswahl des Antibiotikums richtet sich nach einem Erregernachweis und der jeweiligen klinischen Situation. Hier bietet die Leitlinie „Guidelines for the diagnosis, prevention and management of implantable cardiac electronic device infection. Report of a joint Working Party project on behalf of the British Society for Antimicrobial Chemotherapy“ eine exzellente Informationsquelle, u.a. für die geeignete Antibiotikaauswahl [12]. Hierin finden sich klare Empfehlungen und Entscheidungshilfen für verschiedene klinische Szenarien, z. B. mit und ohne Erregernachweis, mit und ohne Aggregatentfernung oder mit und ohne Begleitendokarditis.

Bei schrittmacherabhängigen Patienten kann eine passagere Stimulation mit einer aktiven Elektrode und einem externalisierten Schrittmacher erfolgen [4].

\section{Therapeutische Sonderfälle}

Kleiner Nahtabszess früh nach Implantation: Kommt es zu kleinen, frühen, streng oberflächlichen Infektionen der Taschennaht, kann mit alleiniger Antibiotikatherapie ohne Explantation des Systems verfahren werden, sofern keine Hinweise für eine Tascheninfektion bestehen [13]. Engmaschige Verlaufskontrollen sind erforderlich.

Versuch der Aggregatrettung: Einzelne Patienten können aufgrund eines reduzierten Allgemeinzustands, einer Multimorbidität, eines stark erhöhten Risikos oder einer begrenzten Lebenserwartung für eine Systemexplantation nicht infrage kommen. In diesen seltenen Einzelfällen kann eine Aggregatrettung versucht werden. Hierbei sollte über 6 Wochen eine i.v. Antibiose mit anschließender Verlaufskontrolle und Blutkulturen erfolgen. Ist eine Keimeradikation so nicht möglich, kann auch eine orale Langzeitantibiose diskutiert werden [12]. 


\section{Reimplantation}

Vor einer Reimplantation muss kritisch überprüft werden, ob die ursprüngliche Indikation weiterhin besteht. Bei einem Teil der Patienten wird so eine erneute Implantation verzichtbar, z. B. primärprophylaktische ICD-Implantation mit gebesserter LV-Funktion oder Sick-Sinus-Syndrom mit jetzt permanentem Vorhofflimmern ohne Bradykardien.

Es dürfen keine Infektionszeichen mehr vorliegen und erneute Blutkulturen müssen negativ sein. Die Neuimplantation sollte kontralateral erfolgen. Ist dies nicht möglich, muss auch ein epikardialer Zugang überlegt werden [6,9]. Als Alternativen stehen für diese Fälle auch elektrodenlose Schrittmacher (Leadless Pacer) oder subkutane Defibrillatoren zu Verfügung.

\section{KURZGEFASST}

Neben der Antibiotikagabe ist in aller Regel die komplette Explantation von Aggregat und Elektroden erforderlich. Seltene Sonderfälle sind der kleine Nahtabszess früh nach Implantation und der Versuch der Aggregatrettung.

\section{Fazit}

Die konsequente Prävention von Herzschrittmacher- und ICD-Infektionen durch Kenntnis und Vermeidung von Risikofaktoren ist entscheidend, um Systemexplantationen und langwierige Antibiotikatherapien zu verhindern. Für umfassende Informationen zu diesem Thema empfehlen wir die „Guidelines for the diagnosis, prevention and management of implantable cardiac electronic device infection. Report of a joint Working Party project on behalf of the British Society for Antimicrobial Chemotherapy“ [12].

\begin{tabular}{|c|c|}
\hline ABKİ & NGEN \\
\hline CIED & Cardiac implantable electronic Devices \\
\hline & implantierbarer Kardioverter/Defibrillator \\
\hline & kardiale Resynchronisationstherapie \\
\hline
\end{tabular}

\section{Literatur}

[1] Clémenty N, Carion PL, de Léotoing L et al. Infections and associated costs following cardiovascular implantable electronic device implantations: a nationwide cohort study. Europace 2018. doi:10.1093/europace/eux387

[2] Greenspon AJ, Patel JD, Lau E et al. 16-Year Trends in the Infection Burden for Pacemakers and Implantable Cardioverter-Defibrillators in the United States. J Am Coll Cardiol 2011; 58: 1001-1006

[3] Padfield G], Steinberg C, Bennett MT et al. Preventing cardiac implantable electronic device infections. Heart Rhythm 2015; 12: 2344-2356
[4] Mulpuru SK, Pretorius VG, Birgersdotter-Green UM. Device Infections: Management and Indications for Lead Extraction. Circulation 2013; 128: $1031-1038$

[5] Johansen JB, Jorgensen OD, Moller M et al. Infection after pacemaker implantation: infection rates and risk factors associated with infection in a population-based cohort study of 46299 consecutive patients. Eur Heart | 2011; 32: 991-998

[6] Kirkfeldt RE, Johansen JB, Nielsen JC. Management of Cardiac Electronic Device Infections: Challenges and Outcomes. Arrhythmia Electrophysiol Rev 2016; 5: 183

[7] Markewitz A. Jahresbericht 2015 des Deutschen Herzschrittmacher- und Defibrillatorregisters. Teil 2: Implantierbare Cardioverter-Defibrillatoren (ICD). Herzschr Elektrophys 2018; 29: 100-115

[8] Rizwan Sohail M, Henrikson CA, Jo Braid-Forbes M et al. Increased longterm mortality in patients with cardiovascular implantable electronic device infections. Pacing Clin Electrophysiol 2015; 38: 231-239

[9] Nielsen JC, Gerdes JC, Varma N. Infected cardiac-implantable electronic devices: prevention, diagnosis, and treatment. Eur Heart J 2015; 36: 2484-2490

[10] Unsworth JD, Zaidi A, Hargreaves MR. Increased late complex device infections are determined by cardiac resynchronization therapy-defibrillator infection. Europace 2015. doi:10.1093/europace/euv074

[11] de Oliveira JC, Martinelli M, Nishioka SA et al. Efficacy of antibiotic prophylaxis before the implantation of pacemakers and cardioverter-defibrillators: results of a large, prospective, randomized, double-blinded, placebo-controlled trial. Circ Arrhythm Electrophysiol 2009; 2: 29-34

[12] Sandoe JAT, Barlow G, Chambers JB et al. Guidelines for the diagnosis, prevention and management of implantable cardiac electronic device infection. Report of a joint Working Party project on behalf of the British Society for Antimicrobial Chemotherapy (BSAC, host organization), British Heart Rhythm Society (BHRS), British Cardiovascular Society (BCS), British Heart Valve Society (BHVS) and British Society for Echocardiography (BSE). J Antimicrob Chemother 2015; 70: 325-359

[13] Baddour LM, Epstein AE, Erickson CC et al. Update on Cardiovascular Implantable Electronic Device Infections and Their Management: A Scientific Statement From the American Heart Association. Circulation 2010; 121: 458-477

[14] Kolek M], Patel NJ, Clair WK et al. Efficacy of a Bio-Absorbable Antibacterial Envelope to Prevent Cardiac Implantable Electronic Device Infections in High-Risk Subjects: Efficacy of Bio-Absorbable Antibacterial Envelope. J Cardiovasc Electrophysiol 2015; 26: 1111-1116

[15] Welch M, Uslan DZ, Greenspon AJ et al. Variability in clinical features of early versus late cardiovascular implantable electronic device pocket infections. Pacing Clin Electrophysiol 2014; 37: 955-962

[16] Downey BC, Juselius WE, Pandian NG et al. Incidence and significance of pacemaker and implantable cardioverter-defibrillator lead masses discovered during transesophageal echocardiography. Pacing Clin Electrophysiol 2011; 34: 679-683

[17] Ahmed FZ, James J, Cunnington C et al. Early diagnosis of cardiac implantable electronic device generator pocket infection using 18F-FDG-PET/CT. Eur Heart J Cardiovasc Imaging 2015; 16: 521-530

[18] Erba PA, Sollini M, Conti U et al. Radiolabeled WBC scintigraphy in the diagnostic workup of patients with suspected device-related infections. JACC Cardiovasc Imaging 2013; 6: 1075-1086

[19] Kusumoto FM, Schoenfeld MH, Wilkoff BL et al. 2017 HRS expert consensus statement on cardiovascular implantable electronic device lead management and extraction. Heart Rhythm 2017; 14: e503-e551 